\title{
Power-Law Scaling Regimes for Solid-State Dewetting of
}

\section{Thin Films}

\author{
Rachel V. Zucker, W. Craig Carter, Carl V. Thompson
}

January 27, 2016

\begin{abstract}
The capillary force drives the edges of solid thin films to retract. The distance a film edge has retracted over time is usually fitted to a power law. However, experiments and numerical simulations suggest that edge retraction does not follow a power-law. In this work, a simple geometric model for edge retraction is presented that reproduces the retraction distance versus time scalings of simulations for both isotropic and highly-anisotropic films, and is consistent with experiments. The earliest time at which a power-law fit becomes a reasonable approximation is calculated as a function of substrate-film contact angle.
\end{abstract}

Keywords: dewetting; surface energy; morphological evolution; thin film; capillary forces

Thin films are the fundamental building blocks for many micro- and nano-scale devices and systems. However, they are unstable against capillary forces due to their high surfacearea-to-volume ratio. Capillarity (i.e., surface tension) drives a process in thin films known 
as solid-state dewetting [1], which occurs in the solid state primarily via surface self-diffusion, though other transport mechanisms are possible [2].

The main feature of dewetting is retraction of the film's edges and the formation of thick "rims" of material along the retracting edges. The rim volume primarily comes from the volume of film that has been consumed by the retraction process $[1,3]$. Retraction is facilitated by a mass flux from the receding triple line (the intersection of the film/vapor, vapor/substrate, and substrate/film interfaces) towards the advancing side of the rim [4]. The flux from the bulk film towards the rim is extremely small, and may usually be neglected [5].

Brandon and Bradshaw developed a simple model for edge retraction that provides two important scaling laws [3]. First, the model predicts that the radius of a growing hole in a thin film will increase with time to the $2 / 5$ power. Second, it predicts that the height of the rim will increase with time to the $1 / 5$ power.

The B\&B (Brandon and Bradshaw) model has two major limitations: first, it was developed for a contact angle of $90^{\circ}$ only, and second, the cross-section of the rim was taken to be a semi-circle. Because of the first assumption, the effect of film-substrate contact angle on the scaling is unknown. The second assumption makes the model valid only in the limit of long retraction times, when the rim is much taller than the film height. Other phenomena such as pinch-off $[6,7]$ or fingering instabilities $[8,9,10]$ typically occur on thin film edges, which prevent the system from reaching the long-time limit of edge retraction in many cases.

Experiments do not agree with the scaling predicted by B\&B [1]. For single-crystal nickel thin films, the exponent in the best power-law fit has been reported as 0.4 and 0.56 [11], and $0.38-0.43 \pm 0.1[12]$, for varying crystallographic directions. For single-crystal silicon, the exponent is reported to be $0.42-0.58$ for different film thicknesses and orientations [13], 
and has been fitted by an exponent varying between $1 / 2$ and $2 / 5$ [14].

Numerical simulations of edge retraction also show that the retraction distance does not follow a power law. Both isotropic and fully-faceted films initially retract linearly in time, then the exponent in the power law gradually decreases, approaching $2 / 5$ in the long-time limit $[7,4]$. Kinetic Monte-Carlo simulations give an initial retraction rate proportional to $t^{1 / 2}$, and approaching $t^{2 / 5}$ in the long-time limit [14].

In this work, we identify the underlying physics describing edge retraction which are consistent with experiments and numerical simulations. We present an analytical model, based on Brandon and Bradshaw's approach, which overcomes the limitations of the original model. Our model captures the transition from linear retraction to $2 / 5$ power-law behavior and offers a physical explanation for this phenomenology. The retraction rate and earliest time for $t^{2 / 5}$ retraction are also provided as a function of film-substrate contact angle.

The velocity of an isotropic surface evolving by capillary-driven surface diffusion was given by Mullins as [2]

$$
u_{n}=B\left(\frac{\partial^{2} K}{\partial \sigma_{1}^{2}}+\frac{\partial^{2} K}{\partial \sigma_{2}^{2}}\right)
$$

where $B=\frac{D_{s} \gamma \Omega^{2} \nu}{k T}, D_{s}$ is the surface self-diffusivity, $\gamma$ is the surface energy, $\Omega$ is the volume per atom, $\nu$ is the density of mobile surface atoms, $k$ is Boltzmann's constant, $T$ is temperature, and $K$ is the mean curvature of the surface as a function of orthogonal arc length coordinates $\sigma_{1}$ and $\sigma_{2}$. This equation can be made dimensionless to have the form

$$
v_{n}=\left(\frac{\partial^{2} \kappa}{\partial s_{1}^{2}}+\frac{\partial^{2} \kappa}{\partial s_{2}^{2}}\right)
$$

where $v_{n}$ is the dimensionless velocity and $v_{n}=u_{n} H^{4} / B, \quad H$ is the film thickness, $s_{1}$ and $s_{2}$ are the dimensionless arc length coordinates, $s_{i}=\sigma_{i} / H$, and $\kappa$ is the dimensionless mean 
curvature, $\kappa=H K$.

The following five assumptions made in the B\&B model are preserved in our model to simplify the rim geometry: i) The film is taken to be isotropic. ii) The film edge profile is identical everywhere along the triple line, so there is no dependence on the arc length coordinate parallel to the triple line $s_{2}$, and it can be ignored. iii) When the film is crosssectioned normal to the triple line, the rim profile is a circular arc. iv) The film behind the rim has uniform thickness, i.e., there is no valley ahead of the retracting rim. The discontinuity in slope where the rim meets the film is artificial and is therefore ignored. $v$ ) There is no mass flow between the flat film and the rim.

To overcome the limitations of the original B\&B model, we introduce two augmentations: we allow any contact angle, and perform a more accurate treatment of the rim volume over time. While B\&B take the cross-section of the rim to be a semi-circle, here it is treated as a circle that is cut along two perpendicular chords (see Fig. 1): the horizontal cut ensures that the rim meets the substrate at the equilibrium contact angle $\theta$ (which is no longer constrained to be $90^{\circ}$ ), and the vertical cut ensures a flush match between the rim and bulk film, so that volume is conserved (which was not the case in the original B\&B model).

All lengths in this analysis are normalized to the film thickness $H$, and time is normalized to $H^{4} B^{-1}$, so that all quantities are dimensionless. The height of the rim, $h$, is related to the radius of curvature of the rim, $r$, and the contact angle, $\theta$, by

$$
r=\frac{h}{1-\cos \theta}
$$

To compute the velocity of surface motion using Eqn. 2, the second derivative of curvature along the film profile is needed. We assume that the curvature as a function of arc 
length is parabolic near the triple line, i.e., we use a second-order accurate approximation of the curvature, similar to B\&B's and Danielson's approach $[3,13]$. In general, the second derivative of a parabola, $k(s)$, of best fit to three distinct points $\left(s_{i}, k_{i}\right)$ is

$$
\frac{\partial^{2} k}{\partial s^{2}} \approx \frac{2\left(s_{1} k_{2}+s_{2} k_{3}+s_{3} k_{1}-s_{1} k_{3}-s_{2} k_{1}-s_{3} k_{2}\right)}{\left(s_{1}-s_{2}\right)\left(s_{2}-s_{3}\right)\left(s_{3}-s_{1}\right)} .
$$

Three points are selected along the $s$ coordinate, $\left(s_{i}, k_{i}\right)=\left(0, k_{s=0}\right),\left(\Delta s, k_{s=\Delta s}\right),\left(2 \Delta s, k_{s=2 \Delta s}\right)$, where $\Delta s$ is taken to be the arc length from the triple line to where the rim meets the bulk film,

$$
\Delta s=r\left(\theta+\arcsin \frac{x_{\max }(t)-r \sin \theta}{r}\right),
$$

and the value of $x_{\max }(t)$ is indicated in Fig. 1 . Note that $\Delta s$ is not an infinitesimal quantity, and changes with time. The curvature at the triple line, $k_{s=0}$, is equal to the curvature of the rim, $1 / r$. At arc distance $\Delta s$ and $2 \Delta s$ from the triple line, the curvature $\left(k_{s=\Delta s}\right.$ and $\left.k_{s=2 \Delta s}\right)$ is that of the flat film, 0. Substitution into Eqn. 2 using Eqns. 3 and 5, and projecting the normal motion into the plane of the substrate (i.e., dividing by $\sin \theta$ ), yields

$$
v_{\text {retr }}=\frac{\csc \theta(\cos \theta-1)^{3}}{\left.h^{3}\left(\theta+\arcsin \left(\frac{1}{h} \sin \left(\frac{\theta}{2}\right) \sqrt{2(h-1)(1+h+(h-1) \cos \theta}\right)\right)\right)^{2}} .
$$

The rim height at a future time, $h(t+d t)$, is computed using conservation of mass within the rim. The old rim, with height $h(t)$, will incorporate material from the flat film with a cross-sectional area $d f$, as illustrated in Fig. 1. The cross-sectional area of the rim is found by integrating the curve that describes it from $x_{\min }$ to $x_{\max }$,

$$
\text { rim profile }(x)=\sqrt{r^{2}-\left(r \sin \theta+x_{\min }-x\right)^{2}}-r \cos \theta,
$$

where $x_{\min }=0$ at time $t$ and $x_{\min }=v_{\text {retr }} d t$ at time $t+d t$. The additional volume in the 
rim where $x<x_{\min }$ if $\theta>90^{\circ}$ is also integrated and added. The area of flat film that is incorporated into the rim, $d f$, is simply $\left(x_{\max }(t+d t)-x_{\max }(t)\right)$ (the film thickness is 1$)$.

The cross-sectional area of the film is conserved, giving the equation

$$
\operatorname{rim} \operatorname{area}(t+d t)-\operatorname{rim} \operatorname{area}(t)-d f=0 .
$$

Upon substitution, this equation becomes transcendental, and cannot be used directly to solve for $h(t+d t)$. Therefore, the assumption is made that

$$
h(t+d t)=h(t)+\alpha v_{r e t r} d t,
$$

where $\alpha$ is an unknown quantity. The left-hand side of Eqn. 8 can be linearized by expanding to first order in $d t$. The linearized equation is then solved for $\alpha$, which describes the rim height as a function of time. The expression for $\alpha$ is lengthy, so it is presented as supplementary material. While $\alpha$ does depend on $d t$, the dependence is so weak that $d t$ must be greater than about $10^{7}$ to affect $\alpha$ by a percent. Therefore, removing the $d t$-dependence in $\alpha(d t)$ by setting $d t=1$ (a value of 1 is chosen because $d t$ appears in the denominator of several terms) within the expression for $\alpha$ is a reasonable numerical approximation.

The change in rim height with respect to time is given by

$$
h^{\prime}(t)=\alpha v_{r e t r},
$$

with the initial condition $h(0)=1$. The solution to this equation does not have a closed form, but it can be integrated numerically without specialized algorithms. The numerical solution to $h(t)$ is substituted into Eqn. 6 and numerically integrated to yield the retraction distance of the film edge as a function of time. Using the built-in numerical differential 
equation solver and numerical integration function in Wolfram Mathematica 10 [15] on a ca. 2011 laptop, the total time for the calculation is a few seconds.

A log-log plot of the edge retraction distance versus time is shown in Fig. 2. The dashed guide lines show that initially the slope of the curves is 1 , and at late times, the slope is $2 / 5$. Considering the short and long time limits of these curves allows quantification of the constant of proportionality and the time it takes to transition from $t^{1}$ to $t^{2 / 5}$ retraction behavior.

The growth rate of the rim height is given by Eqn. 10. When the rim height is equal to the film height (time $\rightarrow 0$ ), the retraction velocity simplifies to

$$
v_{\mathrm{retr}, t \rightarrow 0}=-\frac{(\cos \theta-1)^{3}}{\left(\theta^{2} \sin \theta\right)} .
$$

In this same limit, the rate of rim height increase, $\alpha$, goes to zero. Therefore, the rim initially does not grow in height, and the driving force for retraction is unchanged. However, the rim is incorporating mass, so it is growing only in width (along the $x$-axis in Fig. 1 ). With constant driving force, the retraction distance is proportional to the total retraction time,

$$
x(t \rightarrow 0)=-\frac{(\cos \theta-1)^{3}}{\theta^{2} \sin \theta} t .
$$

In the long-time limit, the rim is very large, so $h>>1$. The rim becomes a circular segment with cross-sectional area $(h /(1-\cos \theta))^{2}(\theta-(1 / 2) \sin 2 \theta)$. Setting the rim crosssectional area equal to the swept-up film area, $1 \times x$, gives an expression for the rim height $h$ as a function of retraction distance $x$. The retraction velocity becomes $v_{\text {retr }}=-(\cos \theta-$ $1)^{3} \csc \theta /\left(4 h^{3} \theta^{2}\right)$. Replacing $h$ with the expression for $h(x)$ yields

$$
v_{\text {retr }, t \rightarrow \infty}=\frac{\csc \theta(2 \theta-\sin 2 \theta)^{3 / 2}}{\left(8 \sqrt{2} \theta^{2} x^{3 / 2}\right)} .
$$


Integration results in the retraction distance in the long-time limit going as $t^{2 / 5}$ :

$$
x(t \rightarrow \infty)=\frac{5^{2 / 5}}{2^{9 / 5}}\left(t \frac{(2 \theta-\sin 2 \theta)^{3 / 2}}{\theta^{2} \sin \theta}\right)^{2 / 5} .
$$

This expression is analogous to Brandon \& Bradshaw's result, but generalized for any contact angle, and non-dimensionalized. When $\theta=90^{\circ}$, this expression is identical to Brandon \& Bradshaw's method applied to a straight film edge (the derivation of the straight edge case is provided in the supplementary material, which differs slightly from their original result for a growing hole and from the derivation in Ref. [13]).

For the purposes of fitting experimental data, it is useful to know when the $2 / 5$ power-law model is applicable to within $10 \%$ error for the exponent value, $t_{\text {power-law }}$ (in other words, when the transition from linear to $2 / 5$ power-law behavior is mostly complete). This must be done numerically. Values for $t_{\text {power-law }}$ obtained from this model and from numerical simulations of edge retraction for isotropic and strongly anisotropic materials (Refs. [7] and [4] ) are shown in Table 1. For contact angles between approximately $30^{\circ}$ and $170^{\circ}$, a simple fit can be obtained: the exponent of retraction is less than 0.46 when the dimensionless time is greater than or equal to

$$
t_{\text {power-law }} \approx 10^{5.575-0.0267 \theta}
$$

Eqn. 15 is plotted parametrically as a function of contact angle $\theta$ in Fig. 2. The retraction distance when $t_{\text {power-law }}$ is reached is $3.3 H$ for $\theta=170^{\circ}$, and increases with decreasing contact angle, exceeding $30 H$ for $\theta=45^{\circ}$.

Edge retraction curves for various contact angles are shown in Fig. 3, along with results from Refs. [7] and [4]. These references report on numerical studies of edge retraction for materials with isotropic [7] and fully-faceted [4] surfaces. After non-dimensionalizing, the 
isotropic and fully-faceted simulations give identical retraction curves for a given contact angle, so a single curve represents both data sets. Anisotropy plays no role in determining the edge retraction distance versus time.

Qualitatively, the model (this work) and simulation (Refs. [7, 4]) curves have the same shape, although the model underestimates the retraction rate. This is likely due to the real rim shape having a higher curvature near the triple line than the circular arc approximation. However, $t_{\text {power-law }}$ from the model and simulation curves agree reasonably well, as shown in Table 1.

It is worth noting that pinch-off $[6,7]$ (thinning of the film ahead of the retracting rim) occurs in some material systems, and may lead to film break-up before reaching $2 / 5$ powerlaw behavior. However, the times to pinch-off for isotropic films presented in Ref. [7] are two orders of magnitude larger than $t_{\text {power-law }}$ for a given contact angle, so the power-law regime should be reached before pinch-off.

The model presented here is simple, but it reproduces the main features of dewetting, and gives insight into the underlying cause of the characteristic scalings. Initially, the rim is shaped like a quarter of a circle. As mass is added to the rim, it initially grows rapidly in width, but not height, so the curvature of the rim initially does not change. The driving force for retraction does not change, and retraction proceeds linearly with time. At very late times, Brandon and Bradshaw's [3] assumptions become valid, and the mass swept up is distributed evenly across a rim which is much taller than the film. This geometry leads to the $2 / 5$ power-law scaling.

The linear edge retraction regime is short-lived for contact angles larger than about $90^{\circ}$. This explains why experiments often fail to detect the linear regime. For a typical metal thin 
film heated sufficiently to undergo dewetting at significant rates $\left(H^{4} B^{-1} \approx 10 \mathrm{~s}, \theta \approx 90^{\circ}\right)$, the linear regime lasts only a few seconds. However, it takes $31 / 2$ hours before the exponent in the power-law model is within $10 \%$ of $2 / 5$. Experiments routinely give an exponent fit

that is between 0.4 and roughly $0.6[13,11,14,12]$. Our model suggests that measuring an exponent larger than 0.4 indicates that the transition to $2 / 5$ power-law time regime is not yet complete. Our prediction that edge retraction is not governed by a power law can explain at least some of the discrepancies between experimental best fits and the Brandon and Bradshaw model.

\section{References}

[1] C. Thompson, Ann. Rev. Mater. Res. 42 (2012) 399.

[2] W. Mullins, J. Appl. Phys. 28 (1957) 333.

[3] R. Brandon, F. Bradshaw, Royal Aircraft Establishment Technical Report 66095.

[4] R. Zucker, G. Kim, W. Carter, C. Thompson, C. R. Phys. 14 (2013) 564.

[5] R. Zucker, Ph.D. Thesis, MIT (2015) 1.

[6] D. Srolovitz, S. Safran, J. Appl. Phys. 60 (1986) 255.

[7] H. Wong, P. Voorhees, M. Miksis, S. Davis, Acta Mat. 48 (2000) 1719.

[8] E. Jiran, C. Thompson, J. Elec. Mater. 19 (1990) 1153.

[9] D. Danielson, D. Sparacin, J. Michel, L. Kimerling, J. Appl. Phys. 100 (2006) 083507. 
[10] Y. Fan, R. Nuryadi, Z. Burhanudin, M. Tabe, Japan. J. Appl. Phys. 47 (2008) 1461.

[11] J. Ye, C. Thompson, Acta Mat. 59 (2011) 582.

[12] G. Kim, R. Zucker, J. Ye, W. Carter, C. Thompson, J. Appl. Phys. 113 (2013) 043512.

[13] D. Danielson, Ph.D. Thesis, MIT (2008) 1.

[14] E. Bussmann, F. Cheynis, F. Leroy, P. Muller, O. Pierre-Louis, New J. Phys. 13 (2011) 043017.

[15] W. R. Inc., Mathematica, Wolfram Research, Inc., Champaign, Illinois, 2014. 


\begin{tabular}{|c||c|c|}
\hline$\theta$ & $t_{\text {power-law, this model }}$ & $t_{\text {power-law, simulations }}$ \\
\hline $30^{\circ}$ & 86,000 & 68,000 \\
\hline $60^{\circ}$ & 7100 & 4700 \\
\hline $90^{\circ}$ & 1000 & 830 \\
\hline $120^{\circ}$ & 200 & 190 \\
\hline $150^{\circ}$ & 49 & 88 \\
\hline
\end{tabular}

Table 1: The time at which the power-law model becomes reasonable, $t_{\text {power-law }}$ is tabulated for various contact angles. The values for $t_{\text {power-law, this model were obtained numerically from }}$ the curves shown in Fig. 2. The values for $t_{\text {power-law, simulations }}$ were obtained from Ref. [7] (simulations of edge retraction for an isotropic material) and Ref. [4] (simulations of edge retraction for a fully-faceted material). The values of $t_{\text {power-law }}$ are identical in Refs. [7] and [4], so they appear as a single column here. The times are in units of $H^{4} B^{-1}$. 
(a)
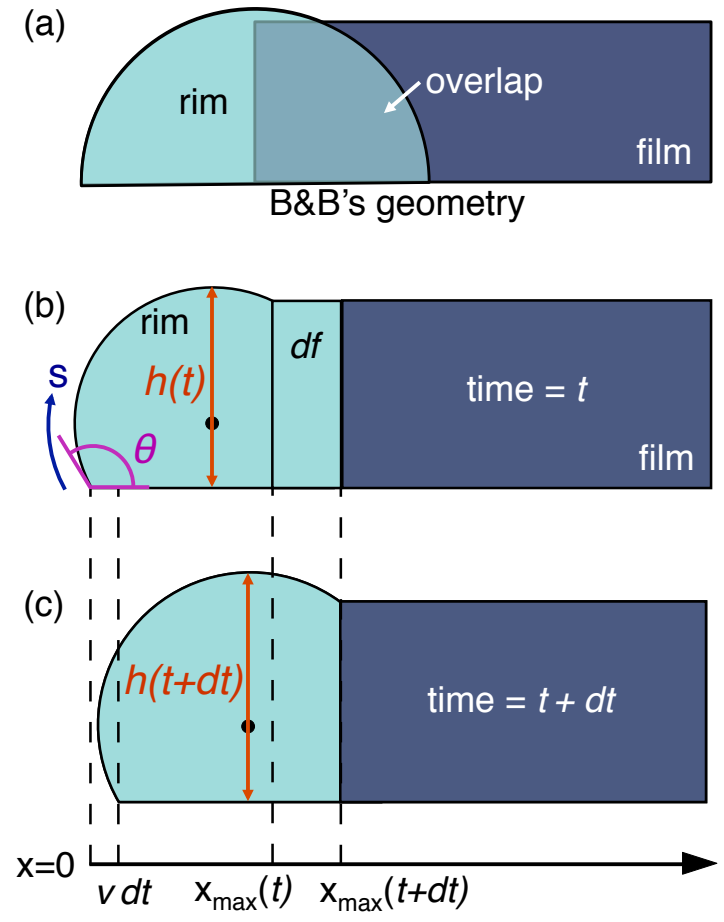

Figure 1: (a) The geometry assumed by Brandon and Bradshaw [3] treats the rim as a semi-circle, with overlap between the film and rim, violating mass conservation. (b, c) The cross-sectional profile of the edge of the film is shown at time $=t(\mathrm{~b})$, and at time $=t+d t$ (c). It is assumed that retraction proceeds at velocity $v$, which is a function of the rim height $h(t)$, for a short amount of time $d t$. The new film edge geometry can be found by assuming that the new rim area (light shading in $(c)$ ) is the sum of the old rim area plus the area $d f$ (light shading in (b) ). The $x$-axis is drawn below the figures, and the positions used in the model are indicated. All length scales are normalized to the film thickness, and the contact angle $\theta$ and arc length coordinate $s$ are shown in (b). 


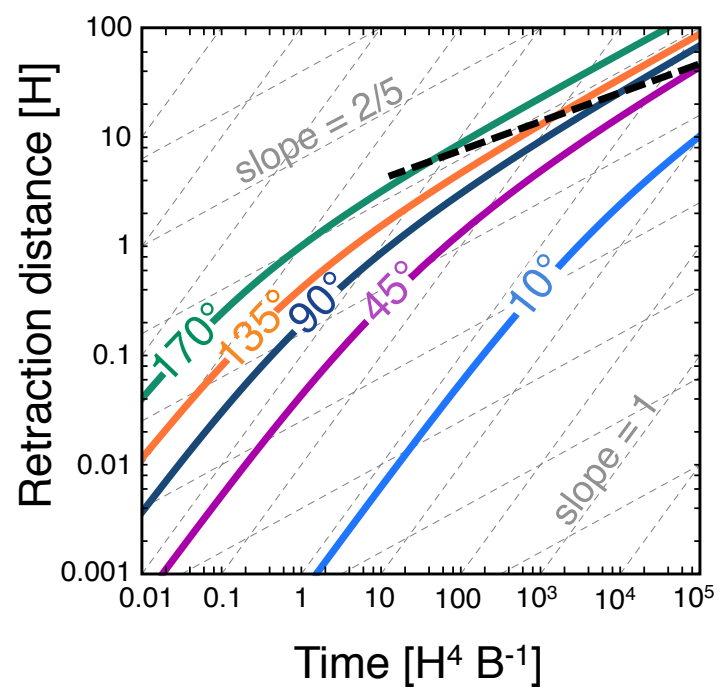

Figure 2: The retraction distance as a function of time is shown for various contact angles. The dashed gray lines are visual guides with slope $=2 / 5$ and slope $=1$. The dashed black line shows the points at which the slope of the retraction curves is equal to 0.46 , given by Eqn. 15. The distance and time are normalized using the film thickness $H$ and material constant $B$. 


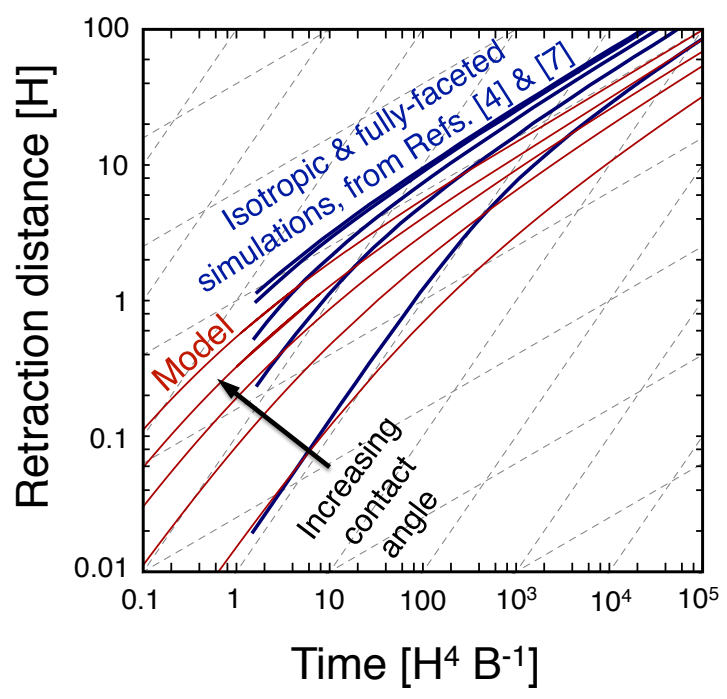

Figure 3: The retraction distance as a function of time for $30^{\circ}, 60^{\circ}, 90^{\circ}, 120^{\circ}$, and $150^{\circ}$ contact angles are shown for the model developed in this work (red), and for simulations of materials with isotropic and fully-faceted surfaces (blue), taken from Refs. [7] and [4], respectively. The dashed gray lines are visual guides with slope $=2 / 5$ and slope $=1$. The isotropic and fully-faceted simulation results are identical, so they appear as a single curve for each contact angle. The model and simulation curves have the same shape, but they are offset. Initially, edge retraction is linear in time, and follows a $2 / 5$ power-law at late times in all three cases. 


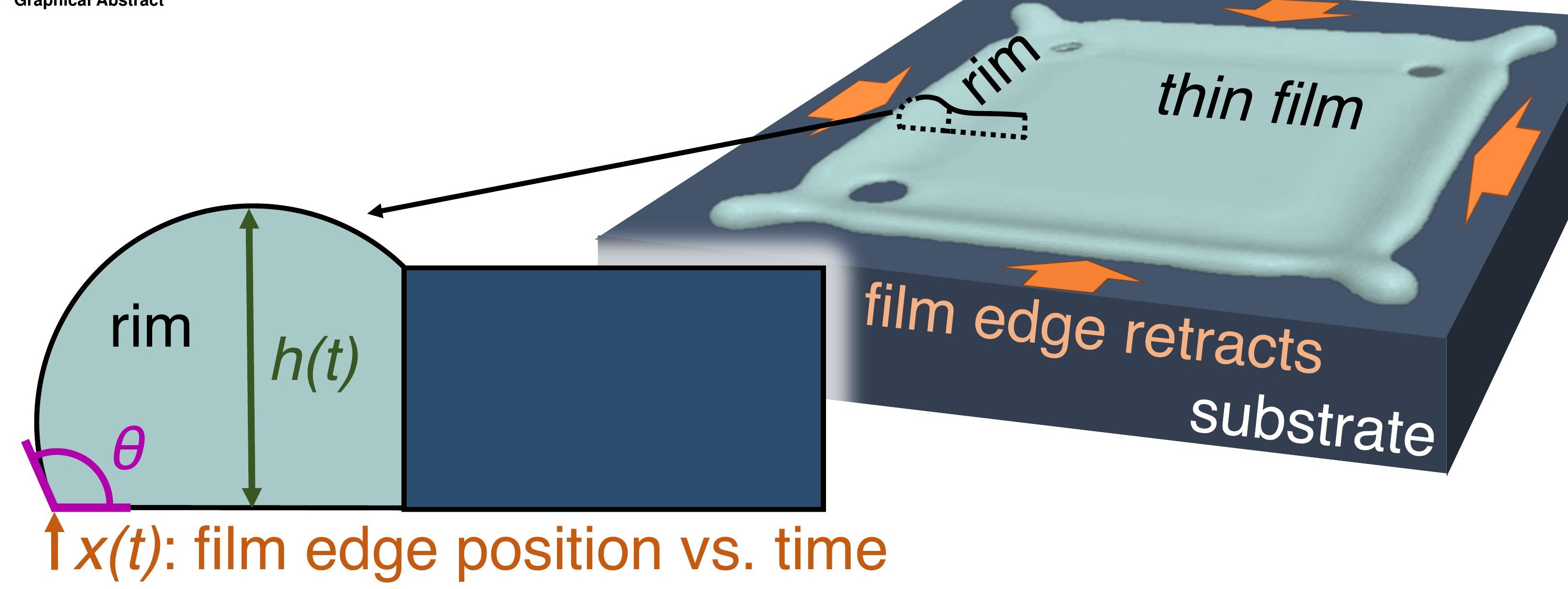

